Check for updates

Cite this: Mater. Adv., 2020, 1, 2339

\section{ZnO nanostructures: a heterogeneous catalyst for the synthesis of benzoxanthene and pyranopyrazole scaffolds via a multi-component reaction strategy $\dagger$}

\author{
Prakash Chhattise, ${ }^{\star a b}$ Suheb Saleh, ${ }^{c}$ Vikram Pandit, (iD b Sudhir Arbuj (iD d and \\ Vasant Chabukswar*a
}

\begin{abstract}
A hydrothermal technique is employed for the preparation of nanostructured $\mathrm{ZnO}$. The reaction was carried out at $180{ }^{\circ} \mathrm{C}$ for $2 \mathrm{~h}$ in the presence of ethylenediamine (EDA) as a capping agent and was characterized by X-ray diffraction (XRD), scanning electron microscopy (FESEM), and transmission electron microscopy (TEM). XRD indicates the formation of highly crystalline ZnO having a wurtzite structure. FESEM analysis validates the formation of a submicron size spherical shaped marigold flower like morphology. The nanostructured $\mathrm{ZnO}$ was screened for its catalytic activity as a heterogeneous catalyst for the multicomponent synthesis of benzoxanthenes and pyranopyrazole derivatives in good to excellent yield. The major advantages associated with this methodology include ease of separation and reusability of the catalyst, easy work-up and short reaction time.
\end{abstract}

Received 10th June 2020

DOI: $10.1039 / d 0 m a 00403 k$

rsc.li/materials-advances

\section{Introduction}

Multi-component reactions (MCRs) play an important role in total synthesis and combinatorial chemistry because of their ability to synthesize heterocyclic scaffolds with numerous degrees of structural diversity. Such reactions have received considerable attention because of their simplicity and economical aspects that allow chemists to explore the synthesis of structurally diverse organic compounds. ${ }^{1}$ The nanostructured metal oxides such as $\mathrm{ZnO}, \mathrm{CuO}, \mathrm{MgO}, \mathrm{TiO}_{2} \mathrm{Fe}_{2} \mathrm{O}_{3}$ etc. have attracted much attention due to their unique properties which offer greater surface-tovolume ratio and higher catalytic activity. ${ }^{2}$

Synthesis of heterocyclic compounds has received substantial attention due to their wide range of pharmacological and biological applications. The benzoxanthenes are a very important class of biologically active heterocyclic compounds in medicinal fields. They exhibit mainly anti-inflammatory, antiviral and antibacterial activities. $^{3-5}$ They can also be used as dyes, ${ }^{6}$ pH-sensitive fluorescent materials for the visualization of bimolecular assemblies ${ }^{7}$ and

\footnotetext{
${ }^{a}$ Department of Chemistry, Ness Wadia Nanomaterial Research Centre, NowrosjeeWadia College (Affiliated to SavitribaiPhule Pune University), Pune, India. E-mail: pk3600se@gmail.com

${ }^{b}$ Department of Chemistry, Haribhai V. Desai Collge, (Affiliated to SavitribaiPhule Pune University), Pune-411002, India

${ }^{c}$ Ministry of Education, Outstanding Secondary School, Iraq

${ }^{d}$ Materials for Renewable Energy Division, Centre for Materials for Electronics Technology, Off Pashan Road, Panchwati, Pune-411008, India

$\dagger$ Electronic supplementary information (ESI) available. See DOI: 10.1039/d0ma00403k
}

laser technologies. ${ }^{8}$ Numerous methods have been reported for the synthesis of benzoxanthene derivatives, which include trontiumtriflate, ${ }^{9}$ silicasulphuricacid, ${ }^{10} \quad \mathrm{P}_{2} \mathrm{O}_{5}$ and indium(III) chloride, ${ }^{11,12}$ methane sulfonic acid, ${ }^{13} \mathrm{NaHSO}_{4}-\mathrm{SiO}_{2},{ }^{14} p$ dodecylbenzenesulfonic acid (DBSA), ${ }^{15,16}$ tetrabutylammoniumhydrogensulphate (TBAHS). ${ }^{17}$ imidazolium based ionic liquid $[$ bmim $] \mathrm{PF}_{6},{ }^{18}$ and trimethylsilyl chloride (TMSCL). ${ }^{19}$ Pyranopyrazole derivatives are a significant class of biologically active heterocycles. They exhibit various biological activities such as anticancer, antimicrobial, analgesic, anti-inflammatory, mollucicidal and fungicidal activities. These derivatives are also used as cosmetics and pigments. ${ }^{20-24}$ Various methods have been reported for the synthesis of these derivatives such as Prolinetriflate, ${ }^{25}$ PTSA in ionic liquid, ${ }^{26}$ magnetic nano particle supported dual ionic liquid 3-sulfobutyl-1-(3-propyltriethoxysilane)imidazolium hydrogen sulfate/ $\mathrm{SiO}_{2}-\mathrm{Fe}_{3} \mathrm{O}_{4} \cdot{ }^{27}$ Nanocrystalline $\mathrm{ZnO},{ }^{28}$ and basic ionic liquids. ${ }^{29}$ Although the traditional synthetic methodologies offer efficiency, most of them suffer from certain limitations such as low yields of the products, use of harsh reaction conditions, toxic solvents and catalysts compared to nanostructured materials. The semiconducting material $\mathrm{ZnO}$ in its nanostructured form has been extensively used in a wide range of technological applications such as electrical engineering, photodegradation of microorganisms, catalysis, and optical and optoelectronic devices. ${ }^{30-36}$ In the present work nanostructured $\mathrm{ZnO}$ is utilized as a heterogeneous catalyst in multi-component organic transformations and can be used for the formation of binary or ternary composite nanomaterials for a variety of applications. ${ }^{37,38}$ In this 
regard, we have developed a simple, efficient, non-toxic heterogeneous catalytic system for the multi-component synthesis of heterocyclic scaffolds like benzoxanthene and pyranopyrazole derivatives via one pot four component reactions.

\section{Experimental}

\section{Materials and reagents}

For the synthesis of $\mathrm{ZnO}$ nanostructures, zinc nitrate $\left(\mathrm{Zn}\left(\mathrm{NO}_{3}\right)_{2}\right.$ $\left.6 \mathrm{H}_{2} \mathrm{O}\right)$, sodium hydroxide $(\mathrm{NaOH})$, and ethylenediamine (EDA) of analytical grade were used as received. All other chemicals, reagents and solvents were of analytical grade and used as received without further purification.

\section{Synthesis of ZnO nanostructures}

For the synthesis of $\mathrm{ZnO}$ nanostructures, $10 \mathrm{mmol}$ of zinc nitrate $\left(\mathrm{Zn}\left(\mathrm{NO}_{3}\right)_{2} \cdot 6 \mathrm{H}_{2} \mathrm{O}\right)$ and $20 \mathrm{mmol}$ of $\mathrm{NaOH}$ were dissolved separately in $15 \mathrm{~mL}$ of distilled water respectively. The $\mathrm{NaOH}$ solution was dropwise added to zinc nitrate solution with constant stirring. To this mixture separately prepared EDA solution $(0.5 \mathrm{~mL}$ in $20 \mathrm{~mL}$ distilled water) was added dropwise and the stirring was continued for $15 \mathrm{~min}$. The whole mixture was then transferred to a stainless steel autoclave containing a $100 \mathrm{~mL}$ Teflon container. The autoclave was sealed properly and heated at $180{ }^{\circ} \mathrm{C}$ for $2 \mathrm{~h}$ in an electric oven. After completion of the reaction the autoclave was naturally cooled down to room temperature. The obtained reaction mass was washed with deionised water in order remove the excess $\mathrm{NaOH}$ and unreacted precursor. Then a final wash was given with ethanol. The sample was dried at $80{ }^{\circ} \mathrm{C}$ in a vacuum oven for $1 \mathrm{~h}$, and the dried sample was ground in a mortar and pestle and used as such for further analysis and to evaluate its catalytic activity study.

\section{Characterization}

The prepared ZnO nanostructures were characterized with different spectroscopic and microscopic techniques. The phases were identified using a powder XRD technique (Bruker AXS model $\mathrm{D}-8,10$ to $70^{\circ}$ range, scan rate $=10^{\circ} \mathrm{min}^{-1}$ ) equipped with a monochromator and Ni-filtered $\mathrm{Cu} \mathrm{K}_{\alpha}(1.5406 \AA)$ radiation. The as synthesised $\mathrm{ZnO}$ nanostructures were studied morphologically using FESEM (HITACHI S-4800). Microstructure analyses were carried out using a field emission transmission electron micrograph (FETEM) performed using a JEOL JEM-2200 FS microscope operating at $200 \mathrm{kV} .{ }^{1} \mathrm{HNMR}$ spectra of organic compounds were recorded on a Varian $400 \mathrm{MHz}$ NMR spectrophotometer with $\mathrm{CDCl}_{3}$ or DMSO- $\mathrm{d}_{6}$ as a solvent. Coupling constants $(J)$ are given in Hertz. Melting points were determined on a Buchi M-560. Mass spectral data were obtained from LC-MS (Shimadzu 2010). The reaction was monitored by thin layer chromatography (TLC) on Merck's silica gel plates $\left(60 \mathrm{~F}_{254}\right)$. Photoluminescence spectra (PL) were recorded by the photoluminescence spectrometer ShimadzuRF-5301.

\section{Synthesis of benzoxanthene}

In a $25 \mathrm{~mL}$ round bottom flask a stoichiometric mixture of $\beta$ naphthol $(1.0 \mathrm{mmol})$, aromatic aldehydes $(1.0 \mathrm{mmol})$ and 1,3 dicarbonyl compound (dimedone) $(1.0 \mathrm{mmol})$ were mixed together with a catalytic amount (5 $\mathrm{mol} \%)$ of $\mathrm{ZnO}$ in the presence of ethanol: water $(5 \mathrm{~mL})$ and refluxed for 1-2 $\mathrm{h}$.

The progress of the reaction was monitored by TLC $(30 \%$ hexane/ethyl acetate). After completion of the reaction, the catalyst was filtered and the resulting product was extracted with ethyl acetate, dried over anhydrous sodium sulfate and the solvent was evaporated under reduced pressure. The crude product was purified by silica column chromatography using hexane/ethyl acetate $(90: 10 \mathrm{v} / \mathrm{v})$ as an eluent. Proton chemical shifts $(\delta)$ are relative to TMS $(\delta=0)$ as an internal standard and expressed in ppm. Coupling constants $(J)$ are given in Hertz. The characterization data, physical constants and NMR of these compounds were found to be identical with those reported in the literature (Table S1, ESI $\dagger$ ).

\section{Synthesis of pyranopyrazole}

In a $25 \mathrm{~mL}$ round bottom flask, ethyl acetoacetate $(1.0 \mathrm{mmol})$, hydrazine hydrate $(1.0 \mathrm{mmol})$, aldehyde $(1 \mathrm{mmol})$, and malononitrile $(1 \mathrm{mmol})$ were mixed together and a catalytic amount ( $5 \mathrm{~mol} \%$ ) of $\mathrm{ZnO}$ was added in the presence of aq. ethanol $(5 \mathrm{~mL})$. The reaction was stirred at room temperature for 15-30 min,

The progress of the reaction was monitored by TLC $(30 \%$ hexane/ethyl acetate). After completion of the reaction, the catalyst was filtered and the resulting product was extracted with ethyl acetate, dried over anhydrous sodium sulfate and the solvent was evaporated under reduced pressure. The crude product was purified by silica column chromatography using hexane/ethyl acetate (Table S2, ESI $\dagger$ ).

\section{Results and discussion}

In order to investigate the crystalline phases, the synthesized $\mathrm{ZnO}$ was analysed with the XRD technique and the XRD pattern is depicted in Fig. 1. The intense XRD peaks confirm the formation of highly crystalline ZnO. The observed XRD peaks at $2 \theta=31.7,34.4,36.2,47.6,56.7$, and $62.8^{\circ}$ are attributed to $(h k l)$ planes (100), (002), (101), (102), (110) and (103) respectively. The peak positions validate the formation of crystalline ZnO having a hexagonal wurtzite phase. XRD matches with the reported ZnO pattern (JCPDS No. 36-1451) indicating high phase purity and crystallinity. The average crystallite size calculated using Scherer's equation is found to be $30 \mathrm{~nm}$. The observed lattice constants of the prepared $\mathrm{ZnO}$ nanostructure having hexagonal wurtzite phase are $a=b=3.24 \AA$ and $c=5.20 \AA$ A. After confirming the crystalline $\mathrm{ZnO}$, further morphological study of a ZnO nanostructure by FESEM and TEM analysis was performed.

\section{FESEM analysis}

The morphological study of the prepared $\mathrm{ZnO}$ nanostructures was carried out using the FESEM technique and the images are presented in Fig. 2. FESEM analysis indicates the formation of 


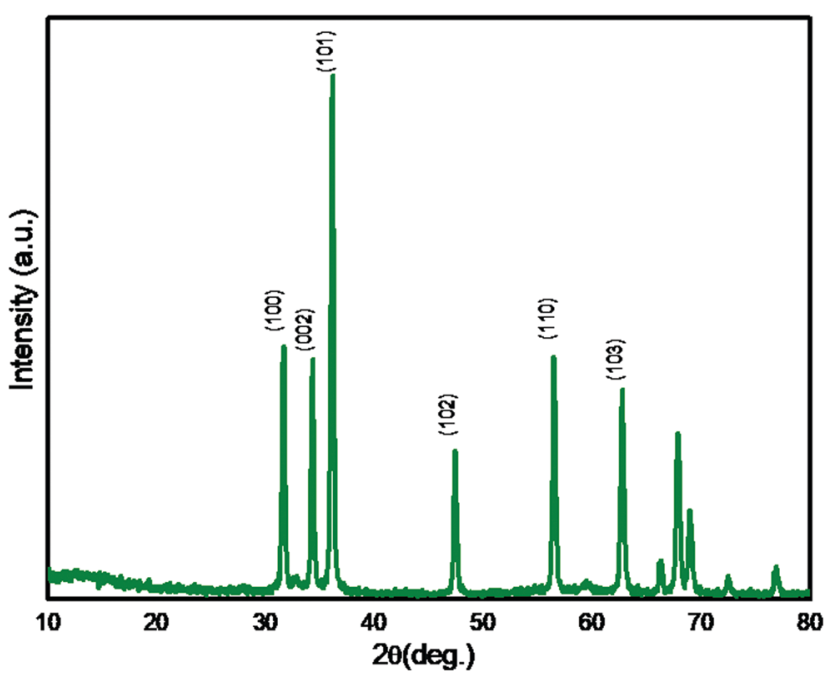

Fig. 1 XRD pattern of $\mathrm{ZnO}$ nanostructures.

submicron sized spherical shape marigold flower like hierarchical morphology having a size in the range of 1 to $1.5 \mu \mathrm{m}$.

This hierarchical $\mathrm{ZnO}$ flower is made up of small nanosized petals having very thin nanosheets of thickness 10-12 $\mathrm{nm}$ and along with the flower like morphology, hexagonal shaped plate and nanorods with 50 to $100 \mathrm{~nm}$ thickness were also observed. But these nanorods and plates are very few as compared to flowers. The use of EDA leads to the formation of a flower like morphology as it forms a complex with zinc ions (Zn-EDA) and restricts the existence of free zinc ions in solution resulting in controlled formation of zinc nuclei. At higher temperature during the reaction the controlled decomposition of this complex leads to the formation of particular shaped morphology; a detailed mechanism study is in progress.
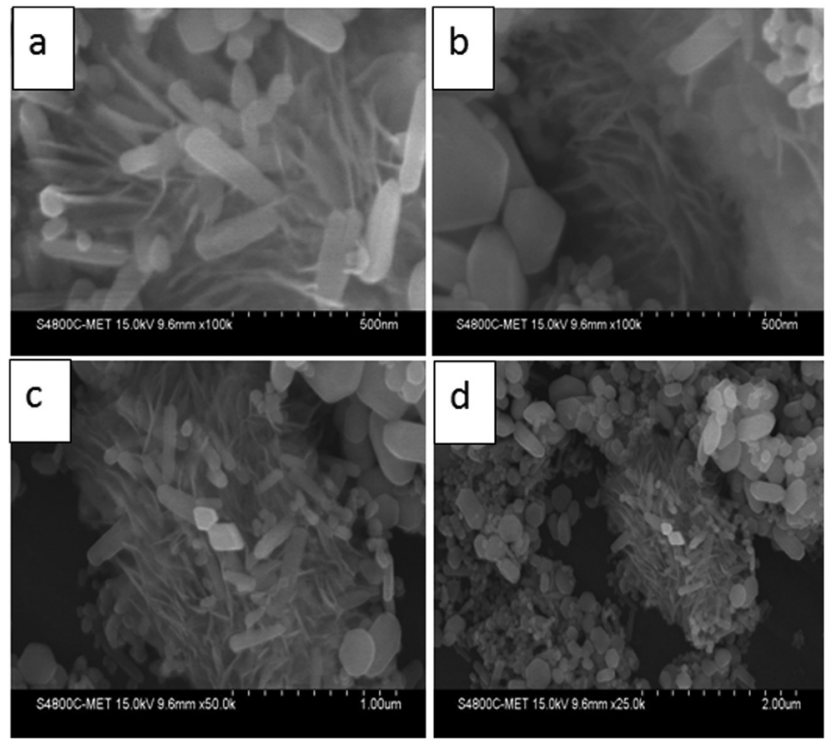

Fig. 2 FESEM of $\mathrm{ZnO}$ nanostructures with different resolutions (reaction conditions $180{ }^{\circ} \mathrm{C}$ for $2 \mathrm{~h}$ ).

\section{TEM analysis}

TEM micrographs of the as synthesized hierarchical ZnO nanostructures along with the selected area diffraction pattern (SAED) are shown in Fig. 3.

TEM micrographs of $\mathrm{ZnO}$ nanostructures prepared at $180{ }^{\circ} \mathrm{C}$ for $2 \mathrm{~h}$ showed formation of hexagonal, spherical and petal like mixed phase morphology. (TEM image a and b). SAED shown in the inset of Fig. 3b indicates the formation of highly crystalline hexagonal wurtzite phase $\mathrm{ZnO}$ nanostructures. The high resolution TEM (HRTEM) image indicates an inter planar spacing of $0.262 \mathrm{~nm}$ corresponding to the [002] plane. From the TEM images it can be seen that the flowers are formed via selfassembling of very thin nanopetals which are created by selfalignment of tiny $\mathrm{ZnO}$ nanostructures. The concentric fused rings can be observed in the SAED pattern and match with the hexagonal phase of $\mathrm{ZnO}$, thereby supporting XRD analysis (inset of Fig. 3b).

\section{Growth mechanism for $\mathrm{ZnO}$ nanostructures}

The growth mechanism for the formation of $\mathrm{ZnO}$ nanostructures is proposed and may follow the following pathways (Scheme 3a-d). During a hydrothermal reaction, the precursors slowly mixed and decomposed into tiny nuclei of $\mathrm{ZnO}$ (Scheme 3a and b). Primarily, tiny nuclei of $\mathrm{ZnO}$ are formed in the supersaturated solution and further growth of nanoparticles takes place with time (Scheme 3c). Under the optimized reaction conditions $\left(180{ }^{\circ} \mathrm{C} /\right.$ hydrothermal, $\left.2 \mathrm{~h} \& \mathrm{EDA}\right)$, the $\mathrm{ZnO}$ nuclei were grown to form hierarchical (hexagonal, spherical and petal like mixed phase morphology) ZnO nanostructures.

The room temperature PL spectrum of the $\mathrm{ZnO}$ nanostructures was obtained with an excitation wavelength of $350 \mathrm{~nm}$ and is shown in Fig. S7 (ESI $\dagger$ ). It shows two distinct peaks at 392 and $490 \mathrm{~nm}$, the sharp and strong ultra violet emission peak at $392 \mathrm{~nm}$ corresponds to band edge emission and the broad emission peak centered at $490 \mathrm{~nm}$ is observed due to oxygen vacancies in the $\mathrm{ZnO}$ lattice. ${ }^{39}$

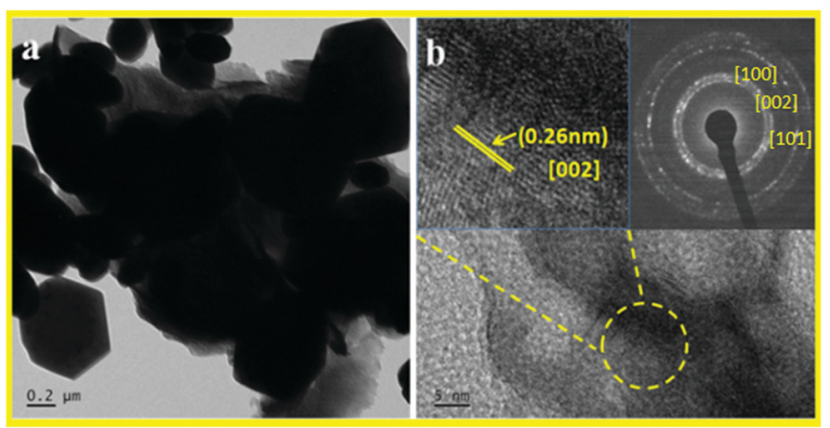

Fig. 3 TEM micrographs of $\mathrm{ZnO}$ nanostructures and SAED image (inset of (b), $180^{\circ} \mathrm{C}$ for $2 \mathrm{~h}$ ). 


\section{Synthesis of benzoxanthene and pyranopyrazole derivatives}

We initially optimized the reaction conditions for the multicomponent reaction of $\beta$-naphthol, benzaldehyde and dimedone to afford the corresponding benzoxanthene derivative. The various reaction parameters comprising amount of catalyst, influence of solvent and temperature were evaluated for the reactions.

\section{Screening of catalyst}

To establish the general reaction conditions for the synthesis of benzoxanthenes, a model reaction of $\beta$-naphthol, $p$-nitrobenzaldehyde and dimedone was studied with and without $\mathrm{ZnO}$ nanostructured material as a catalyst and the results are summarized in Table 1 . The reaction was sluggish and did not proceed in the absence of catalyst even after prolonged reaction time. The use of $\mathrm{ZnO}$ nanostructured material afforded good yield of the desired benzoxanthenes.

In order to find the appropriate concentration of $\mathrm{ZnO}$ for synthesis, the amount of catalyst varied from $0.5 \mathrm{~mol} \%$ to $10 \mathrm{~mol} \%$, and the results are depicted in Table 1 . With increase in catalyst amount the yield of the reaction increases from $65 \%$ to $92 \%$ respectively. With a higher catalyst amount ( $>5 \mathrm{~mol} \%$ ) the yield of the product was around $94 \%$ this indicates that there was not much increase in the yield. The increase in the catalyst amount did not show any significant improvement in the yield of the resultant product. Based on the above observations $5 \mathrm{~mol} \%$ catalyst was used for the synthesis of benzoxanthene derivatives.

\section{Screening of solvent}

The effect of various solvents on the yield of benzoxanthene was also explored using $5 \mathrm{~mol} \% \mathrm{ZnO}$ nanostructured material and the results are summarized in Table 2 .

When the reaction was carried out in water it afforded the desired product in lower yields $(20 \%)$. After extended reaction time this may due to the inhomogeneity of the reactants. It was observed that the yield of benzoxanthenes in aqueous ethanol (50\%) was almost more than $92 \%$ whereas, in methanol it was $85 \%$. Other polar aprotic solvents $\left(\mathrm{CH}_{3} \mathrm{CN}, \mathrm{DCM}, \mathrm{THF}, \mathrm{CHCl}_{3}\right.$, DMF) afforded moderate yield (Table 2, entry 4 to 9), while a non-polar solvent (Table 2, entry 10) resulted in the desired product in lower yield, which required longer reaction time.

Table 1 Optimization of amount of catalyst

\begin{tabular}{lcl}
\hline Entry & ZnO $(x \mathrm{mmol})$ & Yield $^{a}(\%)$ \\
\hline 1 & 0.5 & 65 \\
2 & 1.0 & 78 \\
3 & 2.5 & 85 \\
4 & 5.0 & 92 \\
5 & 7.5 & 94 \\
6 & 10.0 & 94
\end{tabular}

${ }^{a}$ Isolated yield after chromatographic separation. Reaction conditions: aldehyde (1 mmol), 2-naphthol (1 mmol), 1, 3 dicarbonyl compound $(1 \mathrm{mmol})$, catalyst $(5 \mathrm{~mol} \%)$ were stirred in $5 \mathrm{~mL}$ ethanol : water at reflux temperature for $1-2 \mathrm{~h}$.
Table 2 Effect of solvent

\begin{tabular}{|c|c|c|c|}
\hline Entry & Solvent & Time & Yield $^{a}(\%)$ \\
\hline 1 & $\mathrm{H}_{2} \mathrm{O}$ & $6 \mathrm{~h}$ & $>20$ \\
\hline 2 & EtOH : $\mathrm{H}_{2} \mathrm{O}$ & $60 \mathrm{~min}$ & 92 \\
\hline 3 & $\mathrm{MeOH}$ & $90 \mathrm{~min}$ & 85 \\
\hline 4 & $\mathrm{CH}_{3} \mathrm{CN}$ & $60 \mathrm{~min}$ & 89 \\
\hline 5 & DCM & $75 \mathrm{~min}$ & 80 \\
\hline 6 & $\mathrm{THF}$ & $60 \mathrm{~min}$ & 82 \\
\hline 7 & $\mathrm{CHCl}_{3}$ & $60 \mathrm{~min}$ & 86 \\
\hline 9 & $\mathrm{DMF}$ & $90 \mathrm{~min}$ & 65 \\
\hline 10 & Toluene & $120 \mathrm{~min}$ & 55 \\
\hline
\end{tabular}

Among the studied solvents aqueous ethanol (50\%) gave an excellent yield of benzoxanthene within 1-2 h of reaction time. For further investigation we used $5 \mathrm{~mol} \%$ of catalyst and aqueous ethanol (50\%) as a solvent system.

\section{Effect of temperature}

The effect of temperature was studied by carrying out the control reaction at room temperature and under reflux temperature in the presence of nanostructured $\mathrm{ZnO}$ and aqueous ethanol as a solvent. The best result was obtained at reflux temperature $\left(78-80{ }^{\circ} \mathrm{C}\right)$. At room temperature $\left(25-27{ }^{\circ} \mathrm{C}\right)$ the reaction did not proceed even after prolonged reaction time.

To explore the further applicability of this reaction using the optimized reaction conditions, we extended the methodology for the synthesis of diverse derivatives of benzoxanthene using various aromatic aldehydes with either electron-releasing or electron-withdrawing substituents in the ortho, meta and para positions (Scheme 1), and the results are summarized in Table 3.

The benzaldehyde and halogenated benzaldehydes gave almost more than $87 \%$ yield (Table 3, entries $4 \mathbf{a}, \mathbf{4 b}, \mathbf{4 e}, \mathbf{4 i}$ ). 4 -Nitrobenzaldehyde afforded $92 \%$ yield of the corresponding benzoxanthene in a shorter time (Table 3, entry 4c). It was observed that the reaction proceeds at a faster rate with all the aldehydes possessing electron-withdrawing groups on the aromatic ring than aldehydes possessing electron-donating substituents (Table 3, entries $\mathbf{4 d}, \mathbf{4 f}, \mathbf{4 g}, \mathbf{4 h} \& \mathbf{4 j}$ ) giving good to excellent yield of the corresponding products.

After effective application of $\mathrm{ZnO}$ nanostructures as a heterogeneous catalyst for the synthesis of benzoxanthene derivatives we further extended the methodology for multi-component synthesis of pyranopyrazole derivatives. (Scheme 2).

The multi-component reaction of aromatic aldehydes with ethylacetoacetate, hydrazine hydrate and malononitrile in the presence of $\mathrm{ZnO}$ nano structures as a heterogeneous catalyst is
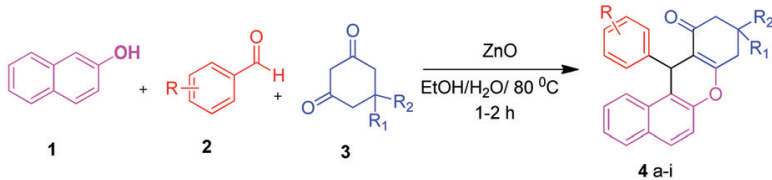

Scheme 1 ZnO catalysed synthesis of benzoxanthene. 
Table 3 Synthesis of benzoxanthenes

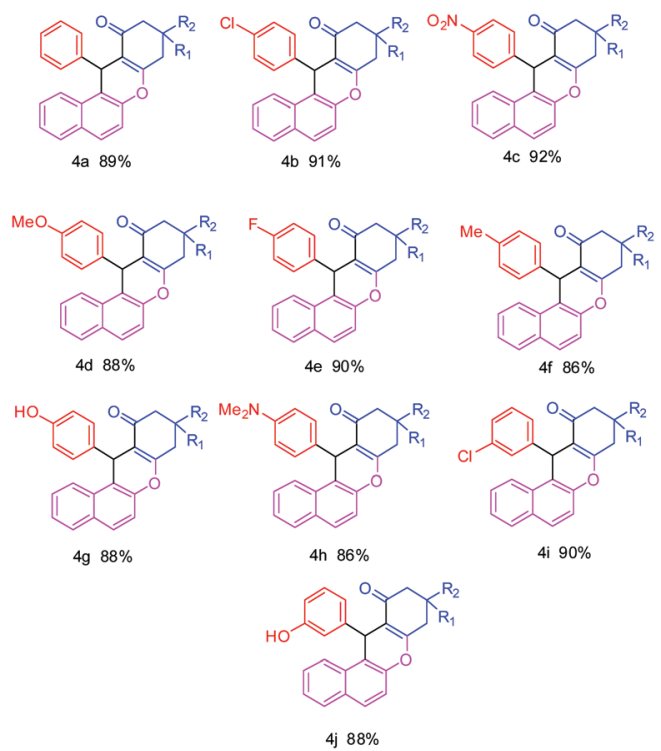

Reaction conditions: Isolated yield after chromatographic separation. Reaction conditions: aldehyde (1 mmol), 2-naphthol (1 mmol), 1,3 dicarbonyl compound $(1 \mathrm{mmol})$, catalyst $(5 \mathrm{~mol} \%)$ were stirred in $5 \mathrm{~mL}$ ethanol-water at reflux temperature for $1-2 \mathrm{~h}$.

described under optimized reaction with respect to the amount of catalyst and solvent as mentioned in Scheme 1.

To optimize the reaction conditions, the model reaction was studied with 4-chlorobenzaldehyde, ethyl acetoacetate, malononitrile and hydrazine hydrate. Various solvents were studied for the optimization of the reaction conditions and the best results were obtained with ethanol-water at room temperature. A wide range of aromatic aldehydes were successfully converted to the analogous pyranopyrazole derivatives under the optimized reaction conditions. It was found that the $\mathrm{ZnO}$ nano structured material effectively catalyzes the reaction within 15-30 min at room temperature affording good to excellent yield of the corresponding derivatives. These results are depicted in Table 4 .

Unsubstituted aromatic aldehyde (benzaldehyde) afforded 90\% yield (Table 4, entry 5a); however amongst the halogenated aldehydes, 4-chlorobenzaldehyde (Table 4, entry 5b) furnished $98 \%$ yield of the resultant pyranopyrazole derivative within $10 \mathrm{~min}$ of reaction time, whereas other halogenated aldehydes afforded more than $90 \%$ yield (Table 4 , entries $\mathbf{5 e}$ and $\mathbf{5 i}$ ). The reaction of 4-nitrobenzaldehyde was completed within 10 min giving 94\% yield of the corresponding product (Table 4, entry 5c). Aldehydes having electron releasing groups also reacted within $15-30 \mathrm{~min}$ with good to excellent yield

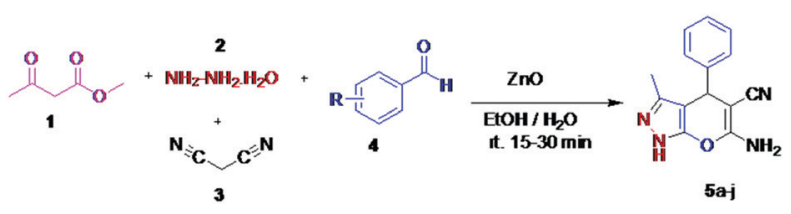

Scheme 2 ZnO catalysed synthesis of pyranopyrazole.

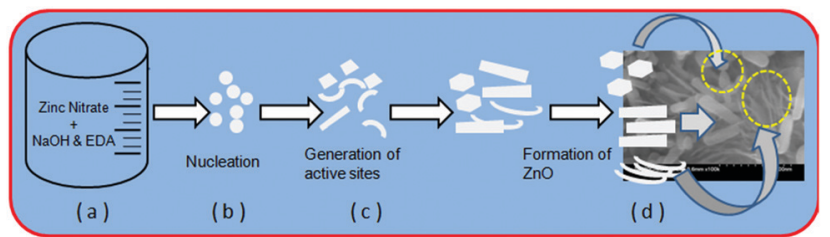

Scheme 3 Plausible growth mechanism of $\mathrm{ZnO}$ nanostructures ((d) is the FESEM image of the $\mathrm{ZnO}$ nanostructures).

Table 4 Synthesis of pyranopyrazole derivatives

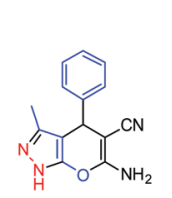

5a $90 \%$
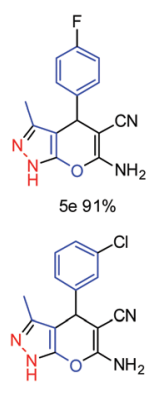

$$
\text { 5i } 90 \%
$$

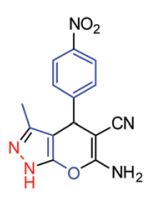

$5 \mathrm{c} 94 \%$
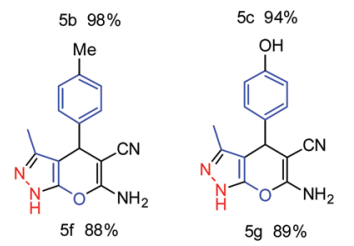

5 g $89 \%$

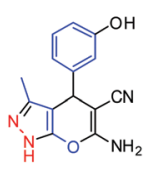

5j $86 \%$

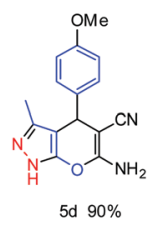

$\mathrm{NMe}_{2}$

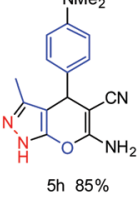

5 h $85 \%$

Reaction conditions: Isolated yield after chromatographic separation. Reaction conditions: ethyl acetoacetate $(1.0 \mathrm{mmol})$, hydrazine hydrate $(1.0 \mathrm{mmol})$, aldehyde $(1 \mathrm{mmol})$, malononitrile $(1 \mathrm{mmol})$ were stirred in ethanol-water for $10-15 \mathrm{~min}$ at room temperature, catalyst (5 mol\%).

(85-90\%) of pyranopyrazole derivatives. (Table 4 entries $5 \mathbf{d}, \mathbf{5 g}$ and $5 \mathbf{j}$ ). It was observed that the majority of reactions proceed at a faster rate with $\mathrm{ZnO}$ nanostructured material at room temperature. Das et al. have reported nano crystalline ZnO (10 mol\%) catalyzed one pot multicomponent reactions for the synthesis of pyranopyrazole derivatives in water: ethanol at room temperature affording 83 to $98 \%$ yield in $3 \mathrm{~h}^{40}$

\section{Recycling study}

The reusability of the catalyst was also studied under the optimized reaction conditions. After completion of the reaction the catalyst was recovered, filtered and washed repeatedly with ethyl acetate and dried.

It was reused under the optimized reaction conditions and it was found that the yields of benzoxanthene and pyranopyrazole were almost comparable. The catalyst recovered after the first cycle was used for the subsequent four cycles and the yield of the corresponding product obtained in each cycle is summarized in Table 5. It was observed that the \% yield of these derivatives was negligibly lowered even after five cycles. These observations suggest that the catalyst surface remains active during successive cycles. Although the slight decrease in the catalytic activity of $\mathrm{ZnO}$ might be 
Table 5 Reusability of the catalyst

\begin{tabular}{lll}
\hline Entry & Number of cycles & Yield $^{a}(\%)$ \\
\hline 1 & Fresh & 95 \\
2 & First & 92 \\
3 & Second & 89 \\
4 & Third & 85 \\
5 & Fourth & 80 \\
$6^{b}$ & Fifth & 94
\end{tabular}

${ }^{a}$ Isolated yield. ${ }^{b}$ Used catalyst from fourth recycle, heated at $150{ }^{\circ} \mathrm{C}$ for 60 min in a $\mathrm{N}_{2}$ atmosphere. Reaction conditions: 1:1:1 mmol reactant with catalyst $(5 \mathrm{~mol} \%$ ) were stirred in $5 \mathrm{~mL}$ ethanol.

due to the deactivation of the active sites of the catalyst. Also, control experimental study of a commercial ZnO sample (bulk size) with as synthesized ZnO nanostructures was carried out. In comparison with the as-synthesized $\mathrm{ZnO}$ nanostructures the commercial $\mathrm{ZnO}$ sample (bulk size) shows 40 to $50 \%$ less reaction yields.

\section{Plausible reaction mechanism for the synthesis of benzoxanthene}

A plausible reaction mechanism for the synthesis of benzoxanthene derivatives using hierarchical $\mathrm{ZnO}$ nanostructures is illustrated in Scheme 4. Hierarchical $\mathrm{ZnO}$ nanostructure coordinate with carbonyl oxygen and enhance the electrophilicity of the carbonyl carbon of aldehydes (1) followed by subsequent attack of dimedone to give an alkene intermediate (2). The nucleophilic attack of an oxygen atom of $\beta$-naphthol (3) to the electron deficient intermediate (2) affords intermediate (4). The subsequent elimination of hydrogen followed by a water molecule (5-6) results in the desired benzoxanthene derivatives (7).

\section{Plausible reaction mechanism for the synthesis of pyranopyrazole}

Ethyl acetoacetate is activated by $\mathrm{ZnO}$ nanostructures followed by attack of hydrazine, which results in the formation of

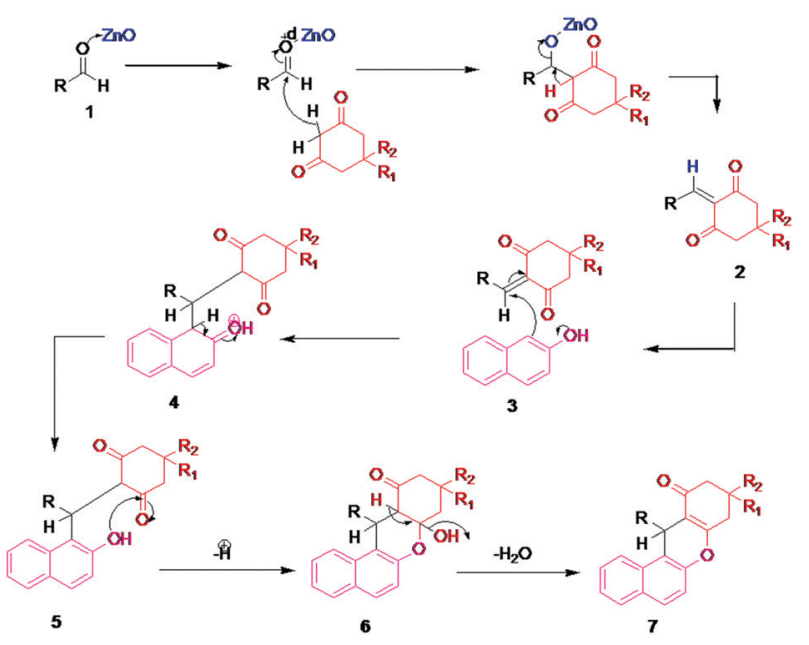

Scheme 4 Proposed reaction mechanism for the synthesis of benzoxanthene derivatives.
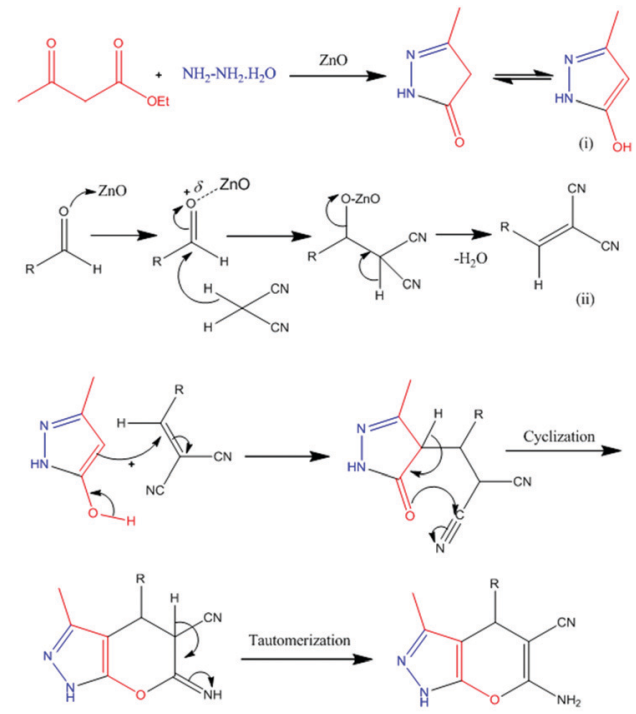

(iii)

Scheme 5 Proposed reaction mechanism for the synthesis of pyranopyrazole derivatives.

pyrazolone, intermediate (i). Also, the carbonyl group of aldehyde is activated by nanostructured $\mathrm{ZnO}$ and then it undergoes Knoevenagel condensation with malononitrile to afford intermediate (ii). The interaction between intermediate (i) and intermediate (ii) enables Michael addition type reaction. The intramolecular cyclization followed by tautomerization gives the corresponding pyranopyrazole derivatives (iii) (Scheme 5).

\section{Conclusions}

In a nutshell, we have successfully used a hydrothermal technique for the synthesis of a highly crystalline hexagonal $\mathrm{ZnO}$ nanostructure having spherical and petal like mixed morphology. Furthermore, the effective use of the synthesized hierarchical $\mathrm{ZnO}$ nanostructures as a heterogeneous catalyst was evaluated for the synthesis of benzoxanthene and pyranopyrazole derivatives via one-pot multi-component reactions in good to high yields under ambient reaction conditions. In addition, the catalyst could be easily recovered by simple filtration and reused for several cycles without significant loss in its catalytic activity.

\section{Conflicts of interest}

There are no conflicts to declare.

\section{Acknowledgements}

The authors sincerely acknowledge Centre for materials for electronics technology (C-MET) Pune and Haribhai V. Desai College, Pune for characterization. We also thank Ness Wadia 
Nanomaterial Research Centre, NowrosjeeWadia College, Pune for providing lab facilities.

\section{References}

1 L. Weber, K. Illegen and M. Almstetter, Synlett, 1999, 366-374.

2 N. Koukbai, E. Kolvari, A. Khazaei, M. A. Zolfigol, B. ShirmardiShaghasemi and H. R. Khavasi, Chem. Commun., 2011, 47, 9230-9232.

3 J. P. Poupelin, G. Saint-Ruf, O. Foussard-Blanpin, G. Marcisse, G. Uchida-Ernouf and R. Lacroix, Eur. J. Med. Chem., 1978, 13, 67-71.

4 R. W. Lambert, J. A. Martin, J. H. Merrett, K. E. B. Parkes and G. J. Thomas, PCT Int. Appl. WO9706178, 1997.

5 T. Hideo and J. Teruomi (Sankyo Co), Japan Pat., 56005480, 1981.

6 S. M. Menchen, S. C. Benson, J. Y. L. Lam, W. Zhen, D. Sun, B. B. Rosenblum, S. H. Khan and M. Taing, US Pat. 6583168, June 24, 2003.

7 C. G. Knight and T. Stephens, Biochem. J., 1989, 258, 683-687. 8 M. Ahmad, T. A. King, D. K. Ko, B. H. Cha and J. J. Lee, J. Phys. D: Appl. Phys., 2002, 35, 1473-1476.

9 J. Li, W. Tang, L. Lu and W. Su, Tetrahedron Lett., 2008, 49, 7117-7120.

10 G. M. Nazeruddin, M. S. Pandharpatte and K. B. Mulani, Indian J. Chem., 2011, 50B, 1532-1537.

11 K. G. Verma, K. Raghuvanshi, R. K. Verma, P. Dwivedi and M. S. Singh, Tetrahedron, 2011, 67, 3698-3704.

12 G. C. Nandi, S. Samai, R. Kumar and M. S. Singh, Tetrahedron, 2009, 65, 7129-7134.

13 Y. B. Shen and G. W. Wang, ARKIVOC, 2008, xvi, 1-8.

14 B. Das, P. Thirupathi, K. Ravinder Reddy, B. Ravikanyh and L. Nagarapu, Catal. Commun., 2006, 737-742.

15 T. S. Jin, J. S. Zhang, A. Q. Wang and T. S. Li, Ultrason. Sonochem., 2006, 13, 220-224.

16 T. S. Jin, J. S. Zang, J. C. Xiao, A. Q. Wang and T. S. Li, Synlett, 2004, 866-870.

17 H. N. Karade, M. Sathe and M. P. Kaushik, ARKIVOC, 2007, xiii, 252-258.

18 M. Kidwai, K. Singhal and S. Kukreja, Can. J. Chem., 2008, 86, 799-802.

19 S. Kantevari, R. Bantu and L. Nagarapu, ARKIVOC, 2006, xvi, 136-148.
20 G. P. Ellis, The Chemistry of Heterocyclic Compounds. Chromenes, Chromanes and Chromones, ed. A. Weissberger and E. C. Taylor, John Wiley, New York, 1977, ch. 11, p. 11.

21 E. A. Hafez, M. H. Elnagdi, A. G. A. Elagemey and F. M. A. A. El-Taweel, Heterocycles, 1987, 26, 903-907.

22 M. A. Sofan, F. M. A. A. El-Taweel and M. H. Liebigs Elnagdi, Ann. Chem., 1989, 935-936.

23 A. Galil, F. B. Riad, S. Sherif and M. Elnagdi, Chem. Lett., 1982, 1123-1126.

24 M. Kidwai, S. Saxena, M. Khan and S. Thukral, Bioorg. Med. Chem. Lett., 2005, 15, 4295-4298.

25 J. Li, L. Lu and W. Su, Tetrahedron Lett., 2010, 51, 2434-2437.

26 J. M. Khurana and D. Magoo, Tetrahedron Lett., 2009, 50, 4777-4780.

27 Q. Zhang, H. Su, J. Luo and Y. Wei, Green Chem., 2012, 14, 201-208.

28 P. Ghosh and A. Das, J. Org. Chem., 2013, 78, 6170-6181.

29 K. Kong, H. L. Wang, D. Fang and Z. L. Liu, Catal. Commun., 2008, 9, 650-673..

30 N. Sakai, G. K. Prasad, Y. Ebina, K. Takada and T. Sakai, Chem. Mater., 2006, 18, 3596-3598.

31 D. Shao, M. Yu, H. Sun, T. Hu, J. Lian and S. Swayer, Nanoscale, 2013, 5, 3664-3667.

32 P. S. Mane, W. J. Lee, H. M. Pathan and S. H. Han, J. Phys. Chem. B, 2005, 109, 24254-24259.

33 N. Madhusudhana, K. Yogendra and K. M. Mahadevan, Res. J. Chem. Sci., 2012, 2(5), 72-77.

34 O. Seven, B. Dindar, S. Aydemir, D. Metin, M. A. Ozinel and S. Icli, J. Photochem. Photobiol., A, 2004, 165, 103-107.

35 D. G. Kumbhar, V. U. Pandit, S. D. Deshmukh, J. D. Ambekar, S. S. Arbuj and S. B. Rane, J. Nanoeng. Nanomanuf., 2015, 5, 227-231.

36 V. U. Pandit, S. S. Arbuj, R. Hawaldar, P. V. Kshirsagar, U. P. Mulik, S. W. Gosavi, C. J. Park and B. B. Kale, J. Mater. Chem. A, 2015, 3, 4338-4344.

37 V. U. Pandit, S. S. Arbuj, U. P. Mulik and B. B. Kale, Environ. Sci. Technol., 2014, 48, 4178-4183.

38 V. U. Pandit, S. S. Arbuj, Y. B. Pandit, S. D. Naik, S. B. Rane, U. P. Mulik, S. W. Gosawi and B. B. Kale, RSC Adv., 2015, 5, 10326-10331.

39 S. S. Arbuj, N. Rumale, A. Pokle, J. D. Ambekar, S. B. Rane, U. P. Mulikand and D. P. Amalnerkar, Sci. Adv. Mater., 2014, 6, 269-275.

40 P. Bhattacharyya, K. Pradhan, S. Paul and A. R. Das, Tetrahedron Lett., 2012, 53(35), 4687-4691. 